\title{
Distribución y abundancia espacial y temporal de Stomolophus meleagris (Rhizostomae: Stomolophidae) en un sistema lagunar del sur del Golfo de México
}

\author{
Francisco Javier Félix Torres ${ }^{1}$, Arturo Garrido Mora ${ }^{1}$, Yessenia Sánchez Alcudia ${ }^{1}$, Alberto de
} Jesús Sánchez Martínez ${ }^{2}$, Andrés Arturo Granados Berber ${ }^{1} \&$ José Luis Ramos Palma ${ }^{1}$

1. Laboratorio de Pesquerías, Centro de Investigación para la Conservación y Aprovechamiento de Recursos Tropicales (CICART). División Académica de Ciencias Biológicas. Universidad Juárez Autónoma de Tabasco. Tabasco, México; francisco7933@gmail.com, garri5609@hotmail.com,jlpalma28@hotmail.com,chin_iita2609@hotmail.com, andres.granados51@hotmail.com

2. Laboratorio de Humedales. Centro de Investigación para la Conservación y Aprovechamiento de Recursos Tropicales (CICART). División Académica de Ciencias Biológicas. Universidad Juárez Autónoma de Tabasco. Tabasco, México; alberthoj.sanchez@gmail.com

Recibido 07-IV-2016. Corregido 04-X-2016. Aceptado 02-XI-2016.

\begin{abstract}
Spatial and temporal abundance and distribution of Stomolophus meleagris (Rhizostomae: Stomolophidae) in a lagoon system Southern Gulf of Mexico. The scyphomedusae feed mainly on microscopic crustaceans, eggs and fish larvae, molluscs and some other jellyfishes. The distribution and abundance of the scyphomedusae has an economic and ecological impact as they are predators that have an influence on the population dynamics of other fisheries. This investigation took place in the lagoon system 'ArrastraderoRedonda', Tabasco, from September 2013 to August 2014, with the purpose to provide information on the distribution, and spatial and temporal abundance of Stomolophus meleagris; along with its relation to environmental parameters. A total of 10 stations were defined and biological samples were taken on a monthly basis during this annual cycle. For this purpose, three pulls with a beach seine monofilament ( $20 \mathrm{~m}$ long by $3 \mathrm{~m}$ height, mesh opening $1.5 \mathrm{~cm}, 5$ to 10 minutes) per station were made within a $1 \mathrm{~km}^{2}$ area. Information obtained was used to calculate the fishing efficiency analysis (CPUE con org./Evaluated Area Unit) and to determine the relationship with the environmental parameters. Our results showed that the abundance in the stations 1 to 3 was from 7 to $19 \mathrm{org} . / \mathrm{km}^{2}$ from April to August 2014; less organisms were observed in stations 6 to 10 (an average of 1 org./ $\mathrm{km}^{2}$ ) from October 2013 to March 2014. The multiple correlation steps analysis indicated that there is a strong association between the CPUE and some environmental investigated variables, and a $70.9 \%$ variability of the data was observed. The salinity resulted the most important variable because it determined a $64.3 \%$ of variation; it was followed by the temperature with a $3.6 \%$; meanwhile, dissolved oxygen explained only the $2.9 \%$, this was mainly because within the area there are several geological, oceanographic and biological factors that favor the distribution of this species due to seawater contribution through the Tupilco bar. With the provided data with this investigation we were able to determine the fishery potential of this resource for Tabasco state. However, additional research is required to allow an extended quantification of this resource, to determine the commercial exploitation areas, and drive studies about reproductive and feeding habits, that may provide the necessary information to establish plans for sustainable use. Rev. Biol. Trop. 65 (1): 167-179. Epub 2017 March 01.
\end{abstract}

Key words: Stomolophus meleagris, distribution, abundance, Tabasco, México.

Los sistemas lagunares estuarinos son de gran importancia debido a que presentan alta tasa de producción primaria y secundaria, además de ser zona de protección, reproducción, crecimiento y alimentación para una gran cantidad de especies marinas, estuarinas y dulceacuícolas (Yáñez-Arancibia, LaraDomínguez, \& Day, 1993; Flores-Verdugo, 2001). Estas características de las lagunas favorecen mayor diversidad de organismos 
meroplanctónicos en la comunidad del zooplancton, en comparación con el holoplancton compuesto por escasas especies (Gasca, Suárez, ECOSUR, \& CONACYT 1996; Odum, 1999). En la región sureste del Golfo de México es una zona con gran potencial pesquero para organismos que tradicionalmente no han sido explotados, ya que en dicha zona se han realizado estudios de organismos como la escifomedusa y el pepino de mar (Omori \& Nakano, 2001; Tunberg \& Reed, 2004). En cuanto al manejo de las pesquerías, actualmente se ignora la importancia que tienen los hábitats, particularmente de los ecosistemas costeros que mantienen a las diversas poblaciones de peces, crustáceos y moluscos entre muchos otros organismos. Por lo tanto, para realizar un buen manejo de los recursos pesqueros del país y en particular la del estado de Tabasco, es primordial conocer y proteger la relaciones entre la productividad de las pesquerías y la permanencia de los hábitats; de esta forma se evita la pérdida del rendimiento del recurso y del desarrollo de los diversos organismos que se encuentran inmersos en estos ecosistemas costeros.

Las medusas son depredadores pelágicos que influyen en la abundancia y distribución de los diferentes grupos de zooplancton, ya sea directamente o por competencia (Purcell, 1992, 1997, 2003; Tunberg \& Reed 2004; Sandin, Walsh, \& Jackson, 2010; De SilvaDávila, Palomares-García, Zavala-Norzagaray, \& Escobedo-Urías, 2006). Por lo tanto, el estudio de este grupo representa un paso en la comprensión de la dinámica de las comunidades del zooplancton marino. Sin embargo, a pesar que son depredadores pelágicos eficientes, que influyen en la abundancia y distribución de los diferentes grupos de zooplancton (ya sea directamente o por competencia), no han sido suficientemente estudiados. Específicamente, no se tiene registros con respecto a la relación que existe entre la presencia de estos organismos y las características ambientales, así como sus aspectos poblacionales, y el efecto de estos organismos en la estructura trófica de los ecosistemas. Las especies de medusas se encuentran con mayor frecuencia en aguas oceánicas, pero muchas especies también habitan zonas estuarinas (Genzano, Mianzán, \& Acha, 2006). Así mismo, desempeñan un papel en el ambiente marino, ya que son indicadores de masas y de corrientes de agua, presentan desplazamientos débiles, además de tener un papel importante como especies depredadoras del plancton. Gómez-Aguirre (1978) menciona que los afloramientos de Stomolophus meleagris han mermado el rendimiento de las pesquerías de peces y camarones del Noreste del Golfo de México, debido la alta depredación (Huang, Christian, \& Colson, 1988) y recomiendan la cosecha de esta especie de medusa, como una forma de proteger las pesquerías. Por su parte, García y Rosenberg (2010), indicaron que la pesca en México es considerada uno de los sectores más importantes para la obtención de proteínas de origen animal, actualmente requeridas por la población. Sin embargo, los efectos de la sobreexplotación a la cual están sometidas las diferentes pesquerías han evidenciado una disminución importante en los volúmenes de captura (FAO, 1997).

La distribución y abundancia de estos organismos del zooplancton gelatinoso se ha estudiado en aguas templadas y subtropicales (Alvariño, 1975, 1999; Arai \& Mason, 1982; Brinton, Fleminger, \& Siegel-Causey, 1986; Zignatti, Cabrera, \& Echaniz, 2012), pero los esfuerzos son relativamente escasos en las zonas tropicales. Además, los pocos estudios sobre la distribución y abundancia de medusas, se han centrado en zonas oceánicas principalmente (Segura, 1984, 1991; Rodríguez-Sáens, Vargas-Zamora, \& Segura-Puertas, 2012). A pesar de que se ha tenido un importante avance en el conocimiento del recurso y su aprovechamiento, existe un desconocimiento sobre la variabilidad espacio-temporal, permanencia y abundancia de $S$. meleagris (López \& Álvarez, 2008), Por lo tanto, para realizar un buen manejo de los recursos pesqueros del país y en particular la del estado de Tabasco, es importante conocer la relación de la abundancia de la especie y su hábitat, y de esta forma tener una idea general sobre la amenaza del rendimiento 
de recurso y favorecer el desarrollo de los diversos organismos que se encuentran inmersos en estos ecosistemas costeros. Adicionalmente, la información necesaria para su correcto aprovechamiento, demanda investigación exhaustiva sobre su biología, distribución y abundancia, parámetros poblacionales y las condiciones ambientales imperantes. Es decir, es indispensable generar toda la información necesaria que permita plantear esquemas de manejo sustentable de los recursos pesqueros. Es por ello que a través del monitoreo en los sistemas lagunares, donde se registre una elevada concentración de escifomedusa, es posible cuantificar los organismos y establecer las zonas potenciales de explotación comercial de este recurso (Graham, Pagès, \& Hammer, 2001). Sin embargo, los efectos locales modifican la respuesta de las comunidades a las variaciones de gran escala temporal o espacial. Es por eso que el presente trabajo tiene como objetivo aportar información sobre la distribución y abundancia espacial y temporal de la escifomedusa, en el sistema lagunar Arrastradero-Redonda.

\section{MATERIALES Y MÉTODOS}

El estado de Tabasco cuenta con una gran diversidad de ecosistemas acuáticos, se encuentra situado entre los $\left(18^{\circ} 12^{\prime} 47^{\prime \prime} \mathrm{N}-94^{\circ}\right.$ 7' 46" W \& $18^{\circ} 39^{\prime} 0.9^{\prime \prime} \mathrm{N}-92^{\circ} 28^{\prime}$ 9" W). Esto es, en la región sureste del país, sobre la llanura costera del Golfo de México, al este con el estado de Campeche y con Guatemala, al sur con el estado de Chiapas y Guatemala, y al oeste con el estado de Veracruz ocupando una superficie territorial de $24455 \mathrm{~km}^{2}$ (INEGI, 1997). Su litoral se extiende a lo largo de 192 $\mathrm{km}$.; su plataforma continental se estima en una superficie de $60 \mathrm{~km}^{2}$ y los sistemas lagunares litorales en 29800 hectáreas. El estado cuenta aproximadamente con 570000 hectáreas de zonas inundables. La gran extensión de las lagunas costeras y el aporte de las aguas de los ríos, ricos en nutrientes, que vierten sus aguas tanto a las lagunas costeras como directamente al mar, originan la formación de ecosistemas de agua salobre, que funcionan como zonas de refugio o protección, reproducción y alimento para los organismos acuáticos, que contribuyen significativamente a la productividad marina costera (SEPESCA, 1984; Contreras, 1988).

La realización del presente trabajo de la distribución y abundancia espacial y temporal de la escifomedusa bola de cañón $S$. meleagris, se llevó a cabo en el sistema lagunar estuarino Arrastradero-Redonda (Fig. 1), del estado de Tabasco, durante los meses de septiembre 2013

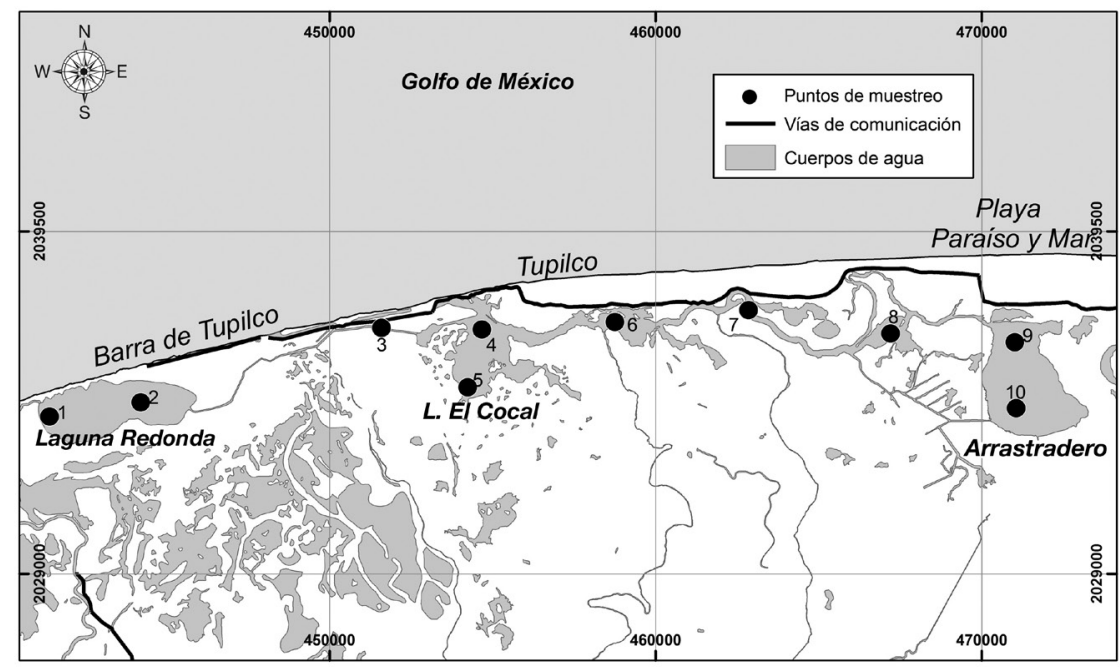

Fig. 1. Área de estudio Arrastradero-Redonda. / Fig. 1. Study area Arrastradero-Redonda. 
a agosto 2014. Se efectuaron recorridos mensuales durante un ciclo anual en 10 sitios de muestreos distribuidos en el sistema lagunar. Se realizaron tres arrastres con un chinchorro playero de monofilamento (de $20 \mathrm{~m}$ de longitud por $3 \mathrm{~m}$ de altura y un luz de malla de $1.5 \mathrm{~cm}$ ) en cada una de las estaciones, con una área de $1 \mathrm{~km}^{2}$, y de una duración de 5 a $10 \mathrm{~min}$.

Muestreo y análisis: Se efectuaron capturas biológicas mensuales por sitios. La distribución y abundancia de la escifomedusa bola de cañón S. meleagris, se analizó a partir de los trabajos de monitoreo. Se realizó el análisis de eficiencia pesquera, expresándose en términos de Captura por Unidad de Esfuerzo (CPUE), se calculó el número de organismos en kilómetros cuadrados (org. $/ \mathrm{km}^{2}$ ), haciendo una evaluación de la representatividad de los resultados obtenidos.

Captura por Unidad de Esfuerzo:

$$
C P U E i j=\frac{W_{i j}}{D i j}
$$

Dónde: $\mathrm{W}=$ Captura, $\mathrm{D}=$ Unidad de superficie evaluada, $\mathrm{I}=$ Sub-área, $\mathrm{J}=$ Estación.

Relaciones entre la abundancia y algunos parámetros ambientales: Se tomaron muestras mensuales durante un ciclo anual de las principales variables ambientales como: temperatura $\left(\mathrm{T}^{\circ} \mathrm{C}\right)$, con un Termómetro UEI PDT300A; la salinidad (S \%o) con un refractómetro marca Atago; oxígeno disuelto $(\mathrm{mg} / \mathrm{L})$ con un Oxímetro Marca YSI DO-200; el pH con un potenciómetro $\mathrm{pH}-100$ marca YSI, y la visibilidad (transparencia) con el disco de Secchi $(\mathrm{cm})$. Se elaboraron cuadros con la información obtenida de manera espacial y temporal en Excel, con el objeto de comparar dichas características en las estaciones y estimar las posibles variaciones de población a lo largo del año. Con la finalidad de establecer las relaciones que existen entre la abundancia de la escifomedusa y los principales parámetros, se efectuó una serie de Análisis de Regresión Múltiple, con el Programa Statgraphic plus 5.1 (Pielou, 1984; Ludwig \& Reynolds, 1988). Para extraer nuevos ejes de variación que resuman la variabilidad de las estaciones de muestreo y su correlación con las variables físico-químicas, se realizó un Análisis de Componentes Principales (ACP) con el paquete estadístico CANOCO 4.5 (Ter Braak \& Smilauer, 2002).

\section{RESULTADOS}

Abundancia espacial y temporal: Los resultados obtenidos del presente trabajo, indicaron que la abundancia en promedio de S. meleagris fluctuaron entre 1 a 9 org. $/ \mathrm{km}^{2}$

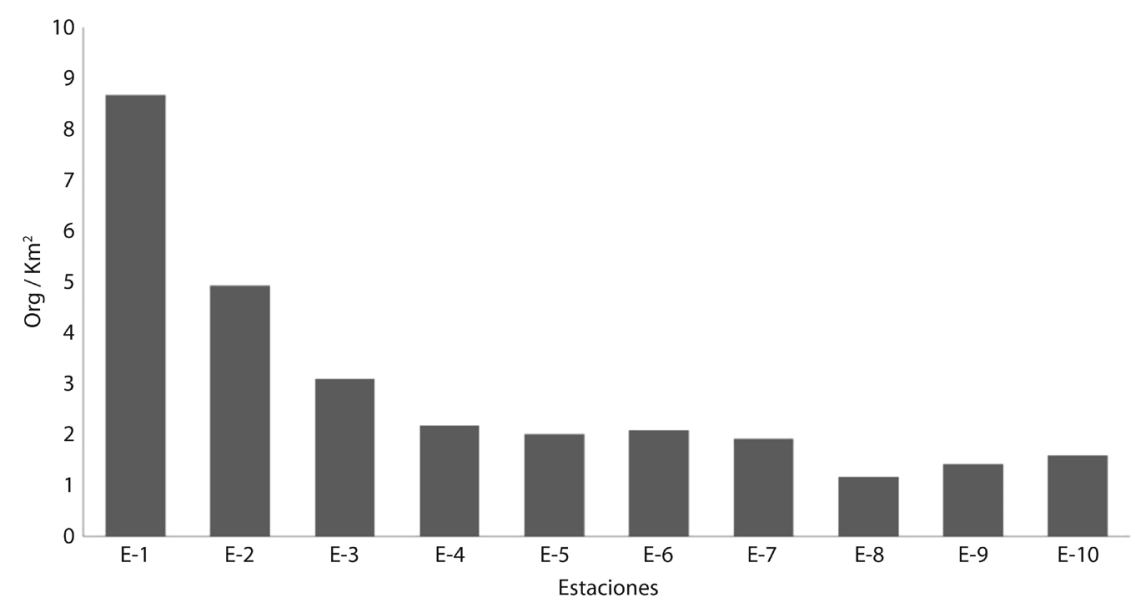

Fig. 2. Abundancia espacial de $S$. meleagris de las estaciones en el sistema lagunar estuarino Arrastradero-Redonda.

Fig. 2. Spatial abundance of $S$. meleagris in stations in the estuary lagoon system Arrastradero-Redonda. 
entre estaciones, y de 1 a 6 org. $/ \mathrm{km}^{2}$ entre meses. Las abundancias más altas se presentaron de la estación 1 a la estación 3, con 19 a 7 org. $/ \mathrm{km}^{2}$ (Fig. 2), principalmente para los meses de abril hasta agosto 2014. Su menor abundancia se observó en las estaciones de la 6 a la 10 , con un promedio de 1 org. $/ \mathrm{km}^{2}$ correspondiente al periodo octubre 2013 a marzo del 2014 (Fig. 3). En el cuadro 1, se muestra las tendencias de los resultados obtenidos, y se observa los datos de la estación 1 a la estación 10 .
Fluctuación de parámetros ambientales: Se tomaron 600 registros de parámetros ambientales durante el ciclo anual de Septiembre 2013 - Agosto 2014. La variabilidad de los factores ambientales evaluados en el sistema, mostró poca variabilidad estacional, asociada a los cambios ambientales de las zonas de muestreo. Se observó que para la temperatura, el mayor promedio fue de $27.6{ }^{\circ} \mathrm{C}$ durante el mes de mayo 2014 y el menor valor promedio fue de $18.2{ }^{\circ} \mathrm{C}$ durante el mes de enero 2014 . Para la salinidad, se observó el valor más alto

\section{CUADRO 1}

Números de organismos de S. meleagris (en promedios) encontrados por cada $\mathrm{km}^{2}$ en el sistema lagunar Arrastradero-Redonda

TABLE 1

Numbers of organisms of $S$. meleagris found (in average) by every $\mathrm{Km}^{2}$ in the lacunose system Arrastradero-Redonda

\begin{tabular}{ccccccccccccc} 
Estación & Sept & Oct & Nov & Dic & Ene & Feb & Mar & Abr & May & Jun & Jul & Agost \\
E-1 & 7.9 & 6.32 & 2.33 & 0 & 0 & 0 & 0 & 8.18 & 13.21 & 10.96 & 8.75 & 14.3 \\
E-2 & 6.32 & 5.53 & 2 & 0 & 0 & 0 & 0 & 5.32 & 6.01 & 5.41 & 6 & 7.4 \\
E-3 & 6.32 & 2.4 & 0.68 & 0 & 0 & 0 & 0 & 2.9 & 2.68 & 4.7 & 6.3 & 6.96 \\
E-4 & 3.95 & 1.37 & 0.75 & 0 & 0 & 0 & 0 & 3.6 & 1.82 & 1.2 & 4.1 & 6.32 \\
E-5 & 0.4 & 3.99 & 0.35 & 0 & 0 & 0 & 0 & 2.16 & 1.76 & 0.79 & 2.8 & 2.42 \\
E-6 & 0.36 & 0.65 & 0.25 & 0 & 0 & 0 & 0 & 2.24 & 2.96 & 1.02 & 2.12 & 3.1 \\
E-7 & 0.4 & 0.4 & 0.11 & 0 & 0 & 0 & 0 & 2.65 & 2.23 & 1.8 & 2.32 & 2.1 \\
E-8 & 0 & 0 & 0 & 0 & 0 & 0 & 0 & 2.14 & 1.84 & 0.66 & 1.4 & 1.97 \\
E-9 & 0.18 & 0 & 0.09 & 0 & 0 & 0 & 0 & 0.92 & 0.76 & 1.5 & 1.6 & 1.1 \\
E-10 & 0.28 & 0.49 & 0.36 & 0 & 0 & 0 & 0 & 1.37 & 0.97 & 0.46 & 1.3 & 0.98 \\
\hline
\end{tabular}

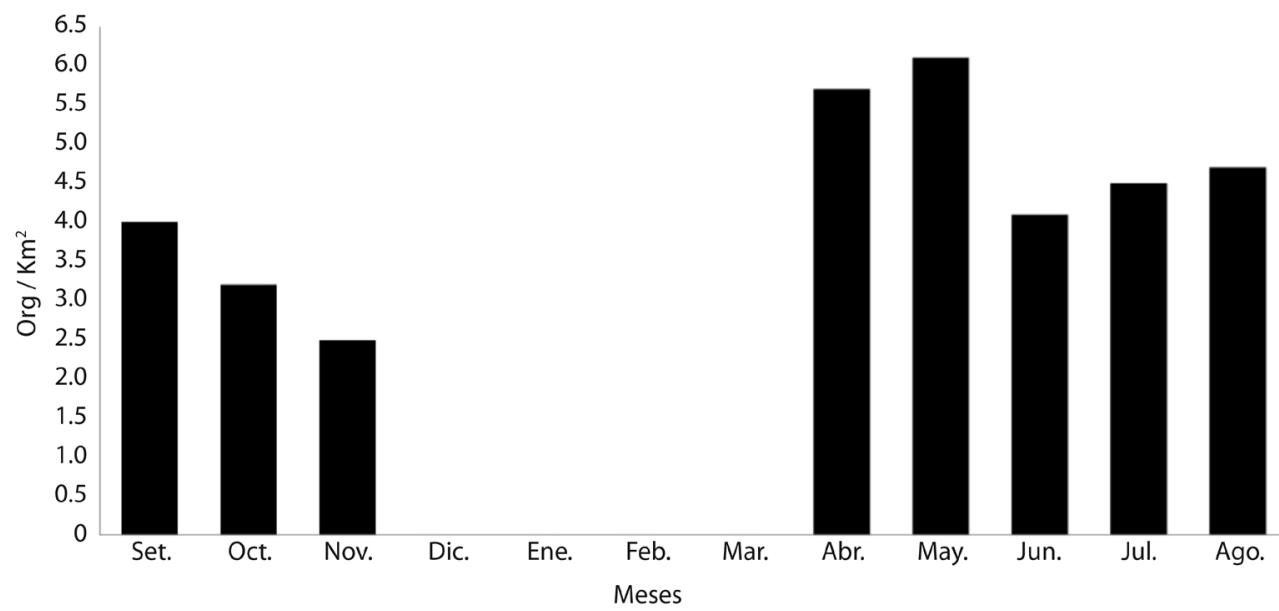

Fig. 3. Abundancia temporal de S. meleagris en el sistema lagunar estuarino Arrastradero-Redonda.

Fig. 3. Temporary abundance of $S$. meleagris in the estuary lagoon system Arrastradero-Redonda. 
de $25.8 \%$ en agosto 2014, y el menor valor promedio de $0.5 \%$ en marzo 2014 . En el caso de la concentración de oxígeno disuelto en este sistema lagunar, se observó que el mayor promedio se presentó durante el mes de septiembre 2013 con $10.1 \mathrm{mg} / \mathrm{L}$, mientras que el menor valor promedio fue de $7.08 \mathrm{mg} / \mathrm{L}$ en julio 2014 (Fig. 4).

Con relación a los valores promedio anuales del $\mathrm{pH}$, el valor más alto fue de 8.3 y se presentó durante el mes de octubre 2013, mientras que el promedio menor fue de 7.0 y se registró durante el mes de julio 2014. Las fluctuaciones de transparencia durante el ciclo anual de muestreo, mostraron que el mayor promedio fue de $61.0 \mathrm{~cm}$ durante noviembre 2013, mientras que el menor valor promedio fue de $39 \mathrm{~cm}$ y se registró en junio 2014 (Fig. 5).

Relaciones entre la abundancia y algunos parámetros ambientales: Se muestra el análisis de componentes principales (ACP), condensando la información contenida en un número grande de variables. La temperatura ${ }^{\circ} \mathrm{C}$ y la salinidad \%o fueron las variables de mayor importancia o de mayor peso. Al realizar el $\mathrm{ACP}$, se encontró que la varianza obtenida al relacionar el componente 1 (variables ambientales) y el componente 2 (CPUE) fue del $74.85 \%$, lo que indica una relación muy alta entre ambos componentes, explicando mejor la variabilidad de los datos. La figura 6 muestra
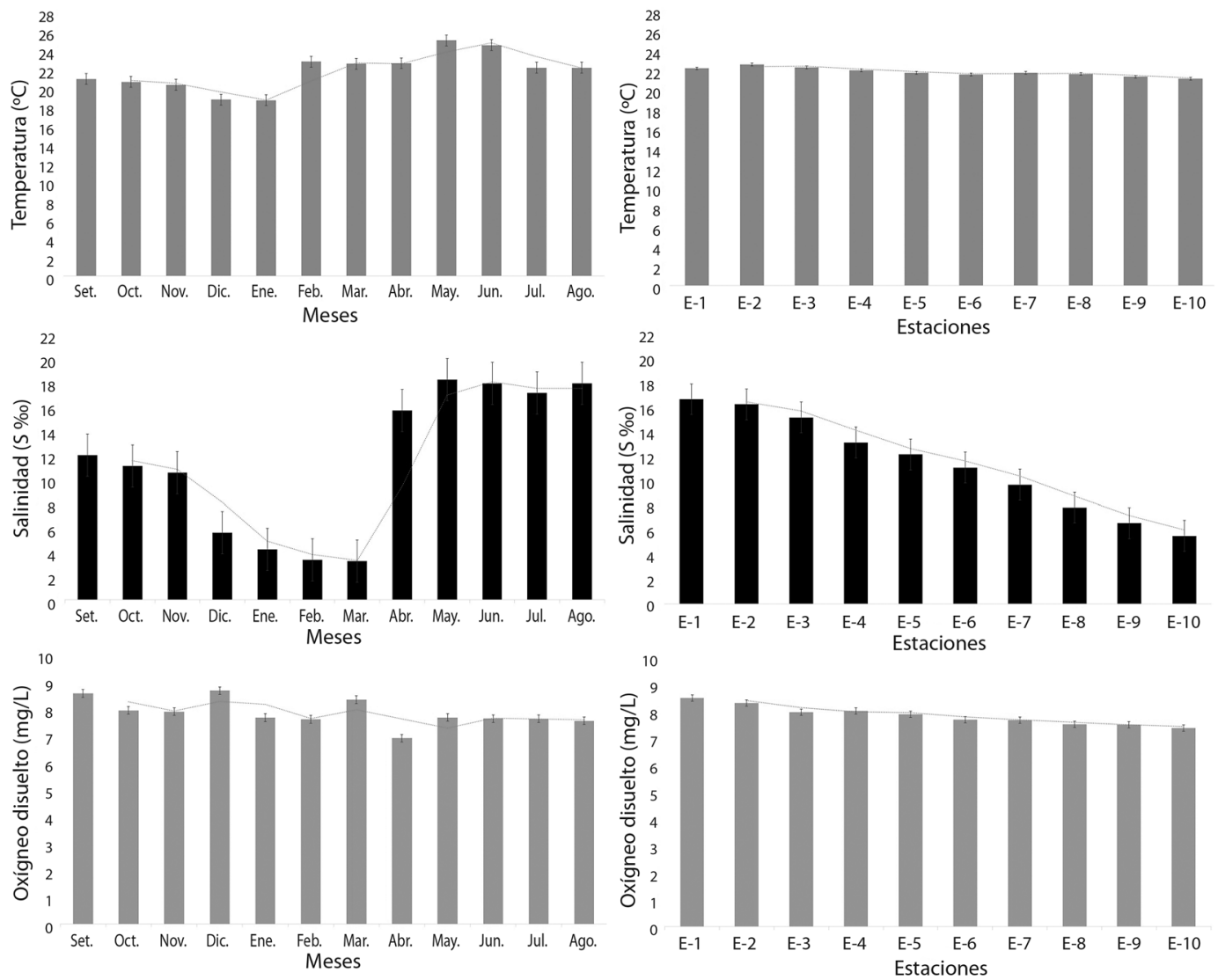

Fig. 4. Fluctuaciones de los valores promedios $( \pm \mathrm{SE})$ de los factores ambientales (temperatura, salinidad y oxígeno disuelto) del Sistema lagunar Arrastradero-Redonda.

Fig. 4. Fluctuations of average values ( \pm SE) of the environmental factors (temperature, salinity, and dissolved oxygen) of the estuary lagoon system Arrastradero-Redonda. 

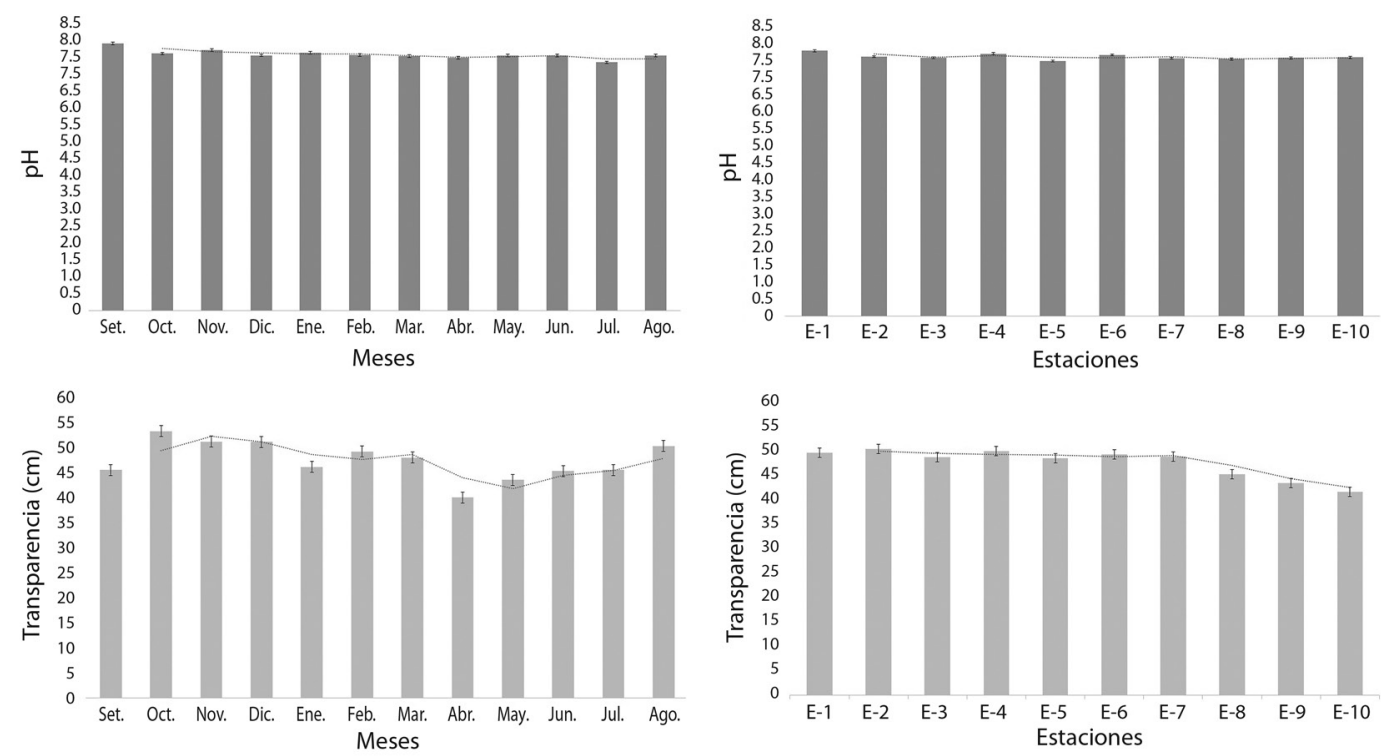

Fig. 5. Fluctuaciones de los valores Promedios $( \pm \mathrm{SE})$ de los factores ambientales (pH y transparencia) del Sistema lagunar Arrastradero-Redonda.

Fig. 5. Fluctuations of average values $( \pm \mathrm{SE})$ of the environmental factors ( $\mathrm{pH}$ and transparency) of the estuary lagoon system Arrastradero-Redonda.

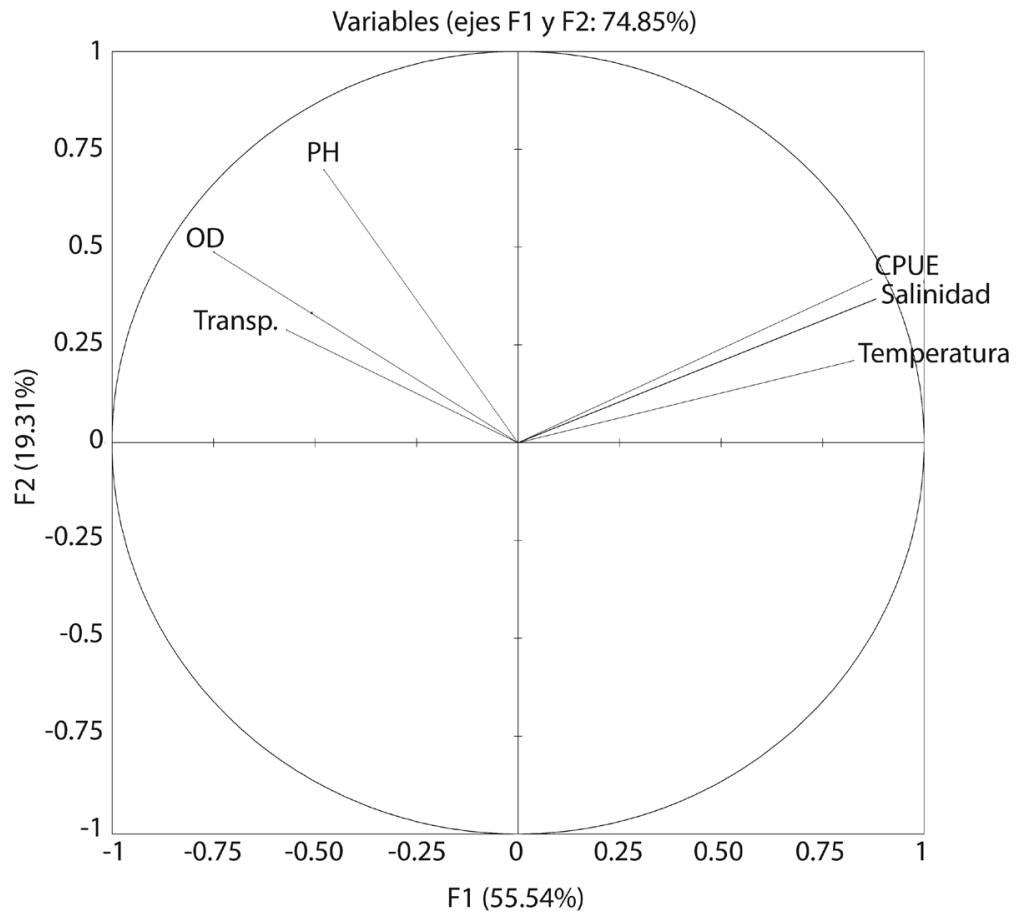

Fig. 6. Analisis de componentes principales de las variables fisicoquímicas (temperatura, salinidad, oxígeno disuelto, $\mathrm{pH}$ y transparencia) del medio, con la CPUE de la escifomedusa $S$. meleagris.

Fig. 6. Analysis of main components of the environmental physicochemical variables (temperature, salinity, dissolved oxygen, $\mathrm{pH}$, and transparency), with the CPUE of the scyphomedusa S. meleagris. 
una clara relación entre la salinidad y temperatura y CPUE de $S$. meleagris en el sistema lagunar Arrastradero-Redonda.

Correlación entre CPUE y los factores ambientales: Al aplicar la prueba de regresión múltiple, fue necesario destacar que ésta necesitaba cumplir con el supuesto de homogeneidad de varianza, esto es, que cada parámetro analizado mostrara una variación definida por un mismo origen, condición que por las características altamente heterogéneas en el comportamiento natural de las variables ambientales en lagunas costeras, dificultó el análisis estadístico mencionado. Por otro lado, la prueba de análisis de varianza llevada a cabo para cada parámetro mostró, en la mayoría de las variables, diferencias significativas en función del cuerpo lagunar, lo que corrobora la particularidad del comportamiento de las variables en cada una de ellas.

El análisis de correlación múltiple indicó que para el periodo muestreado en el sistema lagunar Arrastradero-Redonda, hay una
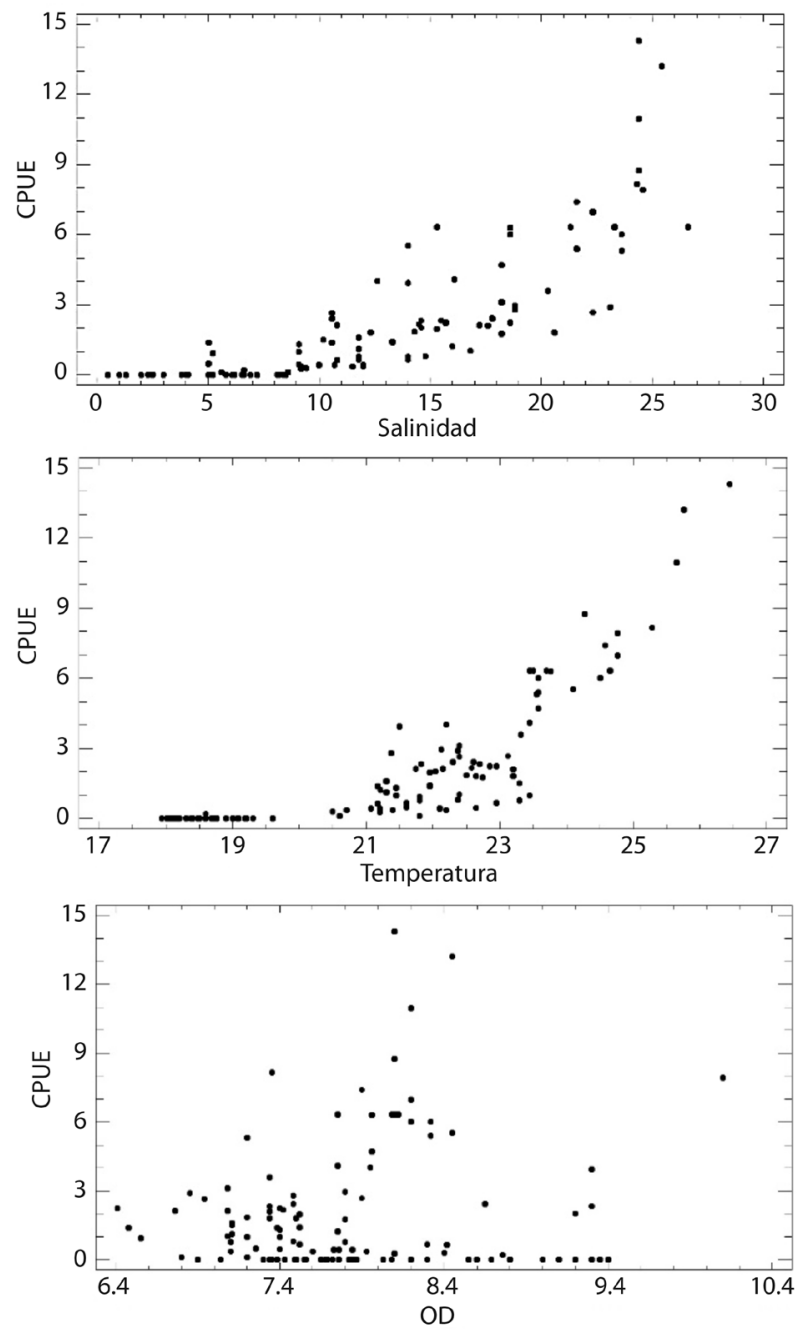

Fig. 7. Análisis de correlación entre Captura por Unidad de Esfuerzo (CPUE) de la escifomedusa (S. meleagris) y las variables ambientales de salinidad, temperatura y oxígeno disuelto.

Fig. 7. Interrelation analysis between scyphomedusa's (S. meleagris) the Catch Per Unit Effort (CPUE) and the environmental variables of salinity, temperature and dissolved oxygen. 
fuerte asociación entre la CPUE y las variables ambientales de salinidad y temperatura. A un $70.875 \%$ de la variabilidad de los datos de CPUE explicada por el modelo. La salinidad fue la variable más influyente, al explicar el $64.31 \%$ de la variabilidad, seguida de la temperatura que explicó un $3.61 \%$, y del oxígeno disuelto, que solo explicó el $2.9 \%$ de la variabilidad de los datos. Las variables de transparencia y $\mathrm{pH}$ no fueron incorporadas al modelo $(\mathrm{P}>0.05)$. La ecuación del modelo ajustado es: $\mathrm{CPUE}=-18.3811$
$+0.618914 *$ Temp_ $+0.138906 *$ salinidad + $0.728787^{*} \mathrm{O} \_\mathrm{D}$. Puesto que el valor-P más alto de las variables independientes fue de 0.0022 , que correspondió a salinidad (y en el cuadro ANOVA fue menor que 0.05 , estadísticamente significativo con un nivel de confianza del 95.0 $\%$ ). La figura 7 muestra la relación entre estas variables ambientales y la variable dependiente; por su parte, la participación de cada variable en el efecto del componente del modelo de regresión obtenido se muestra en la figura 8 .
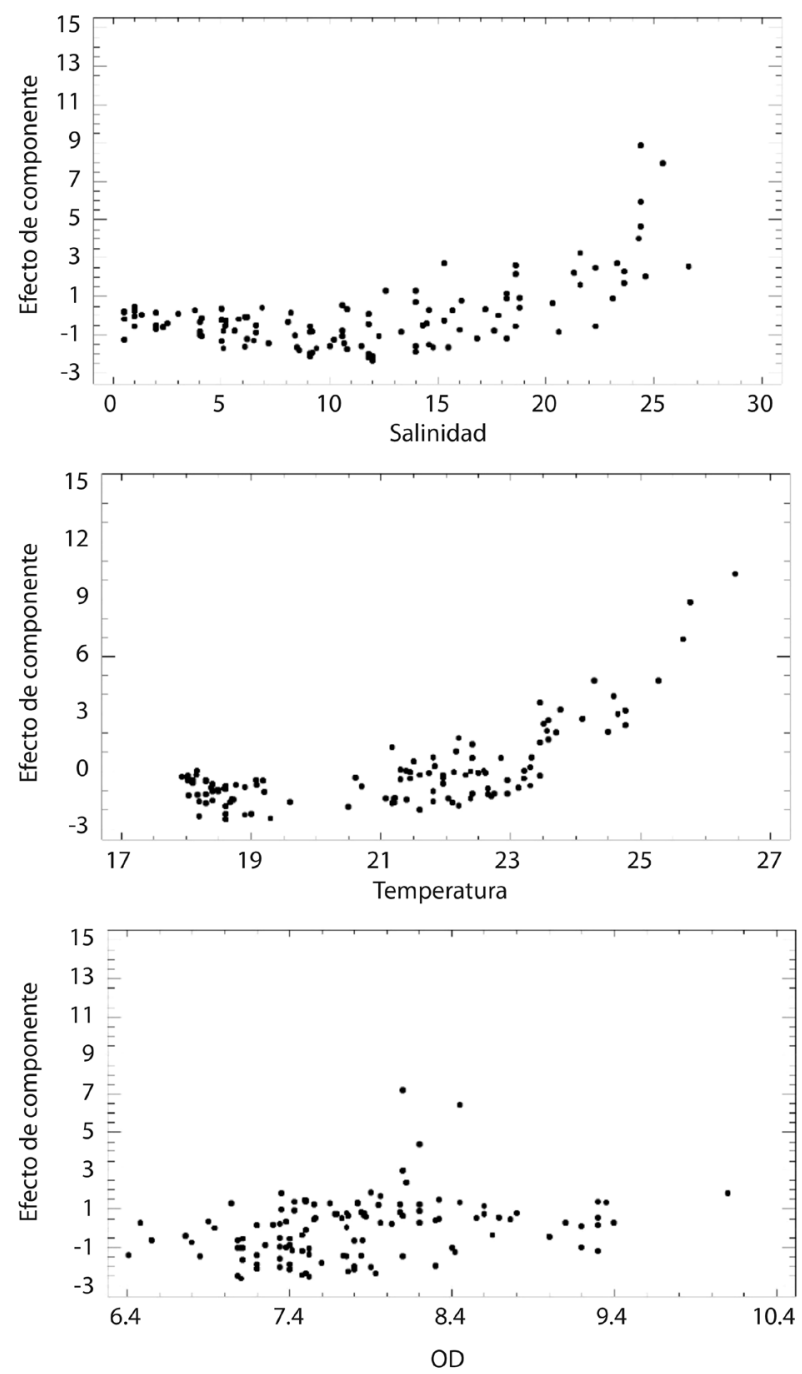

Fig. 8. Interacciones entre las variables ambientales, salinidad, oxígeno disuelto y temperatura, sobre la respuesta o efecto del componente CPUE.

Fig. 8. Interactions between the environmental variables, salinity, dissolved oxygen and temperature on the answers or effect of the CPUE component. 


\section{DISCUSIÓN}

Los resultados obtenidos sobre la distribución y abundancia de la escifomedusa $S$. meleagris durante el ciclo de muestreo (agosto 2013, septiembre 2014) en el sistema lagunar estuarino Arrastradero-Redonda, mostró que un mayor promedio de individuos ocurrió principalmente de la estación 1 a la estación 3 , lo que correspondió principalmente a los meses de abril hasta agosto 2014. Adicionalmente, se observó un patrón de distribución espacial con las abundancias más altas, al presentarse en las zonas con mayor promedio de salinidad y temperatura. Esta zona resultó ser la de mayor influencia de agua marina, principalmente por el aporte recibido a través de la barra de Tupilco. Así mismo, se puede indicar qué otros factores podrían intervenir en la distribución de estos organismos, ya que esta es una especie planctónica que depende casi completamente del movimiento del agua para su desplazamiento, entre ellos, la disponibilidad y distribución del alimento. Este comportamiento en relación con la salinidad es similar a lo reportado por Ocaña y Aguirre, (1999) para las lagunas de Oaxaca, quienes señalaron que la presencia de organismos está íntimamente relacionada con la influencia de agua marina. Asimismo, con lo reportado por Schwartz y Chestnut (1974) que determinaron que la escifomedusa $S$. meleagris fue abundante en zonas con influencias costeras, observándose esporádicamente en diferentes épocas del año. Además Copeland (1965), indicó que la medusa con mayor abundancia fue $S$. meleagris y que se captura durante todo el año, excepto en los meses de julio a septiembre, lo cual sugiere que el movimiento de masas de aguas interviene en la abundancia. Asimismo, Kraeuter y Setzler (1975), observaron que en estas zonas hay una gran variedad de tallas de medusas, incluyendo la $S$. meleagris, desde individuos del estadio de larva, plánula y medusas adultas, y afirmaron que la mayor influencia de tallas adultas se observó en zonas de influencia marina.

En relación con la temperatura, durante el ciclo anual de muestreo de este trabajo, se determinó que la abundancia fue mayor durante los meses con los más altos valores de temperatura de Mayo-Agosto, situación que difiere con lo reportado por Ocaña y Aguirre, (1999), quienes argumentan que a mayor temperatura menor abundancia. Cabe señalar que en relación con este comportamiento, la proliferación de esta especie en el estado de Tabasco, puede atribuirse a la confluencia de condiciones de factores geológicos, oceanográficos y biológicos, en donde la temperatura no representó un factor limitante. Otro de los factores que intervienen fuertemente en la abundancia en este trabajo fue el oxígeno disuelto, ya que las mayores concentraciones se dieron en zonas con abundancias altas, comportamiento similar reportado por Granados y Ramos (1993). En la cual mencionaban que las oscilaciones de oxígeno disuelto para el sistema lagunar del estado de Tabasco eran altas debido a las influencias fluviales; y al ver la regresión lineal, se determinó que explica el $2.3 \%$ de la variabilidad de los datos para dicho sistema lagunar.

Generalmente, un análisis de CPUE empieza con un modelo que considera los posibles efectos externos que intervienen en las abundancias, para luego ir descartando, en pasos sucesivos, los efectos del análisis. Sin embargo, este esquema favorece que los efectos ambientales sean descartados del modelo, cuanto estos efectos no presentan una causalidad con los procesos poblacionales, sino que estos propagan el efecto con algún desfase temporal (Hilborn \& Walters, 1992). Es por eso que, los resultados del análisis multivariado para el periodo muestreado mostraron una fuerte asociación entre la CPUE y las variables ambientales estudiadas, con una regresión positiva. También, destacó que el factor más importante para la abundancia de esta medusa es la salinidad, similar a lo reportado por autores como Ocaña y Aguirre (1999), Mills (2001), Álvarez (2007), Félix (2009), que reportaron una gran abundancia de la escifomedusa bola de cañón $S$. meleagris en zonas con alta salinidades.

Es claro que la determinación de la distribución y abundancia de la escifomedusa $S$. meleagris se ve afectada por la influencia 
de las aguas adyacentes, con una intensidad que varía estacionalmente a lo largo del año. Además, esto concuerda con lo establecido por (Escamilla, Suárez, \& Gasca, 2001) en donde indica que el ingreso de agua marina y las condiciones físico-químicas de un estuario, favorecen la floración de especies como peces, crustáceos y moluscos, así como la del zooplancton, por tanto, en esta zona de influencia marina, se encuentra la fauna residente que coexiste con especies de origen nerítico. La exploración y ubicación de estos recursos con potencial de pesquería, es cada vez más necesaria, con la finalidad de ofrecer alternativas para una diversificación bien dirigida de las actividades pesqueras. Adicionalmente, es indispensable incrementar el conocimiento para su correcto aprovechamiento, y se requiere una investigación exhaustiva sobre su biología, estimaciones de abundancias disponible y explotable, distribución, parámetros poblacionales, impacto de actividades extractivas sobre el ecosistema, en un área más amplia. Es decir, generar toda la información necesaria que permita plantear esquemas de manejo sustentable de los recursos pesqueros (Gómez-Aguirre, 1995).

\section{AGRADECIMIENTO}

La presente investigación no se hubiera podido realizar sin el apoyo en campo de investigadores y compañeros que apoyaron en dicho trabajo. Asimismo, se agradece a Arturo Garrido Mora por sus conocimientos aportados, así como la colaboración para la realización del artículo. Este trabajo contó con el apoyo del Laboratorio de pesquerías de la División Académica de Ciencias Biológicas de la Universidad Juárez Autónoma de Tabasco. Se agradece también al CONACYT por el apoyo de la beca otorgada y al convenio realizado por parte de la Universidad Juárez Autónoma de Tabasco y la Empresa Pesquera México S.A de C.V. para el financiamiento de dicho proyecto.

\section{RESUMEN}

Las escifomedusas se alimentan principalmente de crustáceos microscópicos, huevos y larvas de peces, moluscos y de otras medusas. La distribución y abundancia de la escifomedusa tiene una importancia económica y ecológica ya que son depredadores que influyen en la dinámica poblacional de otras pesquerías. El presente trabajo se realizó de septiembre 2013 hasta agosto 2014 en el sistema lagunar Arrastradero-Redonda, Tabasco, con el objetivo de aportar información sobre la distribución y abundancia espacial y temporal de la escifomedusa Stomolophus meleagris y su relación con los parámetros ambientales. Para su realización se efectuaron recorridos mensuales durante un ciclo anual y se recolectaron muestras biológicas; realizándose tres arrastres en cada una de las estaciones, en una área de $1 \mathrm{~km}^{2}$, con una duración de 5 a $10 \mathrm{~min}$ con un chinchorro playero de monofilamento de $20 \mathrm{~m}$ de longitud por $3 \mathrm{~m}$ de altura y luz de malla de $1.5 \mathrm{~cm}$, con esos datos se realizó el análisis de eficiencia pesquera, (CPUE con Org./Unidad de superficie evaluada) y se determinó la relación con los parámetros ambientales. Se estableció que la mayor abundancia se presentó de la estación 1 a la 3 con 7 a 19 Org $/ \mathrm{km}^{2}$ en los meses de abril hasta agosto 2014 y su menor abundancia se observó en las estaciones 6 a 10 con un promedio de $1 \mathrm{Org} . / \mathrm{km}^{2}$ desde octubre 2013 a marzo 2014. El análisis de correlación múltiple por pasos indicó que existe una fuerte asociación entre la CPUE y algunas variables ambientales estudiadas, observándose el $70.9 \%$ de la variabilidad de los datos. La salinidad fue la variable más influyente explicando el $64.3 \%$ de la variabilidad, seguida por la temperatura con un $3.6 \%$ mientras el oxígeno disuelto explica solo el 2.9 $\%$ de variabilidad, principalmente debido a que en la zona confluyen una serie de factores geológicos, oceanográficos y biológicos favorables para la proliferación de esta especie debido al aporte de agua marina de la barra de Tupilco. Sin embargo falta realizar mayores investigaciones que permita cuantificar este recurso y establecer zonas de explotación comercial y realizar estudios sobre el aspecto biológico, principalmente hábitos reproductivos y alimenticios; que aporten información necesaria para diseñar planes adecuados sobre su posible uso de manera adecuada. Así mismo con los datos obtenidos en el presente trabajo podamos determinar el potencial pesquero de este recurso en el estado de Tabasco.

Palabras clave: Stomolophus meleagris, distribución, abundancia, Tabasco, México.

\section{REFERENCIAS}

Álvarez, T. F. J. (2007). La Pesquería de la Medusa Bola de Cañón (Stomolophus meleagris) en la Región de Bahía de Kino-el Choyudo, Sonora, Durante 2006 (Tesis de Maestría). Instituto Tecnológico de Guaymas, Sonora, Sonora, México.

Alvariño, A. (1975). Los indicadores planctónicos: Distribución batimétrica de algunas especies. In Memorias II Simposio Latino-Americano de Oceanografía Biológica, Cumaná, Venezuela, 1, 139-160. 
Alvariño, A. N. (1999). Hidromedusas: abundancia batimétrica diurna-nocturna y estacional en aguas de California y Baja California, y estudio de las especies en el Pacífico Oriental y otras regiones. Revista de Biología Marina y Oceanografía, 34(1), 1-90.

Arai, M. N., \& Mason, M. (1982). Spring and summer abundance and vertical-distribution of hydromedusae of the central Strait of Georgia, British Columbia. Syesis, 15, 7-15.

Brinton, E., Fleminger, A., \& Siegel-Causey, D. (1986). The temperate and tropical planktonic biotas of the Gulf of California. CalCOFI Reports, 27, 228-266.

Copeland, B. J. (1965). Fauna of the Aransas Pass Inlet, Texas. I. Emigration as shown by tide trap collections. Publications of the Institute of Marine Science, 10, 9-21.

Contreras, F. (1988). Las lagunas costeras mexicanas. México DF, México: Centro de Ecodesarollo, Secretaria de Pesca.

De Silva-Dávila, R., Palomares-García, R., Zavala-Norzagaray, A., \& Escobedo-Urías, D. C. (2006). Ciclo anual de los grupos dominantes del zooplancton en Navachiste, Sinaloa (pp. 26-39). In M.E. Hendrickx (Ed.), Contributions to the study of East Pacific crustaceans 4(1). Instituto de Ciencias del Mar y Limnología, Universidad Nacional Autónoma de México, Sinaloa, México.

Escamilla, J. B., Suárez, M, E., \& Gasca, R., (2001). Distribución del zooplancton durante flujos de marea opuestos en el complejo lagunar de Chelem, Yucatán, México. Revista de Biología Tropical, 49(1), 47-51.

FAO. (1997). Ordenación pesquera. FAO Orientaciones Técnicas para la Pesca Responsable (No. 4). Roma: FAO.

Félix, T. F. J. (2009). Aspectos ecológicos de la medusa bola de cañón (Stomolophus meleagris) en ecosistemas lagunares estuarinos del estado de Tabasco, México. (Tesis de maestría). Universidad Juárez Autónoma de Tabasco, Villahermosa, Tabasco.

Flores-Verdugo, F. J., (2001). Capítulo 6: Procesos Ecológicos en Humedales. En: F. J. Abarca y M. Herzig (eds.). Manual para el manejo y conservación de los humedales en México. Game \& Fish Department of Arizona, RAMSAR, NAWCC, DUMAC, Pronatura, F \& W Service US, SWS, Semarnat y Env. Canada, pp. 1-26.

García, S. M., \& Rosenberg, A. A. (2010), Food security and marine capture fisheries: characteristics, trends, drivers and future perspectives. Philosophical Transactions of the Royal Society of London B: Biological Sciences, 365(1554), 2869-80.

Gasca, R., Suárez, E., Colegio de la Frontera Sur (Chetumal, México), \& Consejo Nacional de Ciencia y
Tecnología (México). (1996). Introducción al estudio del zooplancton marino. México, D.F., México: El Colegio de la Frontera Sur (ECOSUR), Consejo Nacional de Ciencia y Tecnología.

Genzano, G. H., Mianzan E. M., \& Acha, E. G. (2006). First record of the invasive medusa Blackfordia virginica (Hydrozoa: Leptomedusae) in the Río de la Plata estuary, Argentina-Uruguay. Revista Chilena de Historia Natural, 79(2), 257-61.

Gómez-Aguirre, S. (1978), Ingresos masivos de Stomolophus meleagris Agassiz (Scyphozoa-Rhizostomeae) en lagunas costeras de México. Memoria. I Congreso Nacional de Zoología, 9, 114-124.

Gómez-Aguirre, S. (1995). Cnidaria de México: Rasgo histórico y diagnostico preliminar de su estudio. Resúmenes. VI congreso latinoamericano de ciencias del mar. 95.

Graham, W. M., Pagès, F., \& Hamner, W. M. (2001). A physical context for gelatinous zooplankton aggregations: a review. Hydrobiologia, 451, 199-212.

Granados, A. A. \& Ramos, J. L. (1993). Comportamiento de la salinidad, temperatura, oxígeno disuelto y $\mathrm{pH}$ superficial de un sistema lagunar costero del estado de Tabasco México. In Res. V Congreso Latinoamericano de Ciencias del Mar. 247.

Hilborn, R., \& Walters, C. J. 1992. Quantitative fisheries stock assessment. New York: Chapman and Hall.

Huang, Y., Christian, P., \& Colson, D. (1988). Harvest and preservation of shrimp by catch: cannonball jellyfish (Stomolophus meleagris). Proceedings. XII Annual Conference of the Tropical and Subtropical Fisheries Technological Society of de Americas. University of Florida, Gainesville, E.U.A.

Instituto Nacional de Estadística, Geografía e Informática (INEGI). (1997). Anuario estadístico del estado de Tabasco. Aguascalientes: INEGI.

Kraeuter, J. N., \& Setzler, E. M. (1975). The seasonal cycle of Scyphozoa and Cubozoa in Georgia estuaries. Bulletin of Marine Science, 25(1), 66-74.

López, M. J. \& Álvarez, T. J. (2008). Medusa bola de cañón: recurso de exportación. Ciencia y Desarrollo, $34,8-15$.

Ludwig, J. A., \& Reynolds, J. F. (1988). Statistical ecology: a primer in methods and computing (Vol. 1). John Wiley \& Sons.

Mills, C. E. (2001). Jellyfish blooms: are populations increasing globally in response to changing ocean conditions? In Jellyfish Blooms: Ecological and Societal Importance (pp. 55-68). Netherlands: Springer.

Ocaña, G., L., \& Aguirre, S. G. (1999). Stomolophus meleagris (Scyphozoa: Rhizostomeae) en dos lagunas 
costeras de Oaxaca, México. Anales del Instituto de Biología. Serie Zoología, 70(2), 71-77.

Odum, E. P. (1999). Ecología. México DF, México: Interamericana.

Omori, M. \& Nakano, E. (2001). Jellyfish fisheries in Southeast Asia. Hydrobiologia, 451, 19-26.

Pielou, E. C. (1984). The interpretation of ecological data: a primer on classification and ordination. New York: John Wiley \& Sons.

Purcell, J. E. (1992). Effects of predation by the scyphomedusan Chrysaora quinquecirrha on zooplankton populations in Chesapeake Bay, USA. Marine Ecology Progress Series, 87, 65-76.

Purcell, J. E. (1997). Pelagic cnidarians and ctenophores as predators: selective predation, feeding rates, and effects on prey populations. Annales-Institut Oceanographique, 73(2), 125-138.

Purcell, J. E. (2003). Predation on zooplankton by large jellyfish, Aurelia aurita, Cyanea capillata and Aequorea aequorea, in Prince William Sound, Alaska. Marine Ecology Progress Series, 246, 137-152.

Rodríguez-Sáenz, K., Vargas-Zamora, J., \& Segura-Puertas, L. (2012). Medusas (Cnidaria: Hydrozoa) de una zona de afloramiento costero, Bahía Culebra, Pacífico, Costa Rica. Revista de Biología Tropical, 60(4). doi:http://dx.doi.org/10.15517/rbt.v60i4.2177

Sandin, S. A., Walsh, S. M., \& Jackson, J. B. (2010). Prey release, trophic cascades, and phase shifts in tropical nearshore ecosystems. Trophic cascades: chapter 5: predators, prey, and the changing dynamics of nature, $453,71-90$

Segura-Puertas, L. (1984). Morfología, sistemática y zoogeografía de las medusas (Cnidaria--Hydrozoa y Scyphozoa) del Pacífico tropical oriental
(Publicación especial 8: 1-320). México, D. F: Instituto de Ciencias del Mar y Limnología, Universidad Nacional Autónoma de México.

Segura-Puertas, L. (1991). Medusas (Cnidaria: Hydrozoa y Scyphozoa) de la región del Domo de Costa Rica. Revista de Biología Tropical, 39(1), 159-163. doi:http://dx.doi.org/10.15517/rbt.v39i1.24846

SEPESCA. (1984). Catálogo de Especies Acuáticas de importancia comercial en el Estado de Tabasco, Artes y Métodos de Captura. Secretaria de pesca y Gobierno del estado de Tabasco.

Schwartz, F. J., \& Chestnut, A. F. (1974). Biological investigations of noxious coelenterates and ctenophores in coastal North Carolina. Morehead City, N.C.: Division of Commercial Fisheries, North Carolina Department of Natural and Economic Resources.

Ter Braak, J. F., \& Smilauer, P. (2002). CANOCO Reference Manual and User's Guide to CANOCO for Windows: Software for Canonical Community Ordination (Microcomputer Power, Ithaca, NY). Ithaca, NY, USA.

Tunberg, B. G. \& Reed, A. S. 2004. Mass occurrence of the jellyfish Stomolophus meleagris and an associated spider crab Libinia dubia, Eastern Florida. Florida Scientist, 67, 94-104.

Yáñez-Arancibia, A., Lara-Domínguez, A. L., \& Day, J. W. J. (1993). Interactions between mangrove and seagrass habitats mediated by estuarine nekton assemblages: Coupling of primary and secondary production. Hydrobiologia, 264(1), 1-12.

Zignatti, A., Cabrera G., \& Echaniz, S. (2012). Changes in the zooplankton and limnological variables of a temporary hypo-mesosaline wetland of the central region of Argentina during the drying. Pan American Journal of Aquatic Sciences, 7(2), 93-106. 
